\title{
Introduction of the EU Digital COVID Certificate in Slovenia: Technological and Process Aspects
}

\author{
Dalibor STANIMIROVIC ${ }^{\mathrm{a}, 1}$ and Lucija TEPEJ JOCIC ${ }^{\mathrm{a}}$ \\ ${ }^{a}$ National Institute of Public Health, 1000 Ljubljana, Slovenia
}

\begin{abstract}
In early 2021, the European Commission presented a proposal to introduce an EU Digital COVID Certificate, which should enable safe border crossings for citizens within the EU during the COVID-19 pandemic. Subsequently, all EU Member States successfully introduced the EU Digital COVID Certificate by 1 July 2021. This article focuses on a review of the technological and process aspects identified in the introduction of the EU Digital COVID Certificate in Slovenia. The research applies a case study framework, including focus group discussions, as the primary data collection method. The research findings expose the technological and process complexities related to the dispersed data sources and fairly intricate and copious business rules used for the creation of the EU Digital COVID Certificate. Moreover, the study implies that the ad hoc introduction of such demanding and sensitive digital solutions in the future will not be possible without the establishment of effective national health information infrastructures across the EU.
\end{abstract}

Keywords. COVID-19 pandemic, EU Digital COVID Certificate, technological and process aspects, case study, Slovenia

\section{Introduction}

The EU Digital COVID Certificate (DCC) was introduced on 1 July 2021 in all EU Member States and it is based on the uniform EU framework for the issuance, verification, and acceptance of interoperable certificates of COVID-19 vaccinations, tests, and recovery, which are intended to facilitate free movement under the pandemic health conditions [1]. The DCC is recognized in all EU Member States, which generally enables the harmonized elimination of potential travel restrictions, and above all ensures the same approach to issuing and in particular verifying DCCs. All EU citizens and third-country nationals legally residing in the EU who hold a DCC should be exempt from restrictions on free movement while traveling in the same manner as nationals of the EU Member States visited. However, regardless of the uniform arrangement of and agreement on the DCC, EU countries will still be able to introduce measures independently to a certain extent [2], which means that the conditions for free movement may still differ between countries. The DCC contains essential information such as name and surname, date of birth, date of issue, relevant information on the vaccination against COVID-19, negative test results, and recovery from COVID-19, and a unique certificate identifier. The DCC

1 Corresponding Author, Dalibor Stanimirovic, Centre for Healthcare Informatics, National Institute of Public Health, Trubarjeva Street 2, 1000 Ljubljana, Slovenia; E-mail: dalibor.stanimirovic@nijz.si. 
is only issued for COVID-19 and the countries visited may not keep this information. Only the validity and authenticity of the DCC are verified, and the authority that issued and signed the DCC is checked. The health information remains the responsibility of the Member State that issued the DCC.

In Slovenia, the entire DCC system was established by the National Institute of Public Health (Nacionalni Inštitut za Javno Zdravje, hereinafter: NIJZ), which also manages the national eHealth solutions and is the only authorized DCC issuer in the country. The digital solution enabling the creation of the DCC is based on the records contained in the Central Registry of Patient Data (CRPD) and provides access to the DCC for patients, healthcare professionals, and authorized personnel at the NIJZ. The DCC can be stored in paper form or on a mobile device. Both versions are equipped with a QR code that contains the essential information and a digital stamp, which attests to the authenticity and validity of the DCC. This article focuses on a review of the technological and process aspects identified in the introduction of the DCC in Slovenia.

\section{Methods}

A case study framework, including focus group discussions as the primary data collection method, was applied to review the technological and process aspects identified in the introduction of the DCC in Slovenia. The choice of the research framework was based on the specifics of the research topic [3], and the approach used was deemed to be the most reasonable one.

Following a comprehensive examination of the related literature and EU recommendations and guidelines, an extensive analysis of the introduction of the DCC in Slovenia was carried out with the focus group participants. NIJZ experts in charge of the introduction of the DCC in Slovenia, representatives from the Ministry of Health, and experts from external IT providers participated in twenty focus group sessions conducted from April to July 2021 (each teleconference session lasted approximately two hours). The expertise and experience of the fifteen prominent experts in the field participating in the focus group discussions (excluding medical expert groups dealing with the epidemiological concerns) ensured the credibility of the research findings. The insights and opinions of the participating experts were supported by the data and reports from the statistical and business intelligence modules contained in the DCC Application and other eHealth solutions. The views and discussions from the focus group sessions were documented in writing. A conventional content analysis was used for the analysis of the data obtained [4], and, along with the literature and EU materials, provided a means to review the technological and process aspects identified in the introduction of the DCC in Slovenia.

\section{Results}

A new eHealth solution, i.e. the DCC Application, ensures the issuance of the DCC in accordance with uniform EU standards. It is based on the CRPD, which is the fundamental source of data and provides essential functionalities for the DCC Application. Data sources for the DCC are records of nucleic acid amplification test (NAAT) and rapid antigen test (RAT) results, as well as records of COVID-19 vaccinations. The DCC is available immediately upon the submission of the respective 
test or vaccination record. A request for a DCC is triggered each time a common CRPD document query is performed, and the DCC is added to the patient's other healthcare documents. As a result, all healthcare providers in Slovenia have immediate access to the DCC for every patient via existing local hospital information systems (and there is no need to upgrade these systems at points of care). Alternatively, healthcare professionals can obtain the DCCs of patients via the zVEM+ web solution, which is aimed at those healthcare providers whose information systems are not yet connected to the CRPD (e.g. pharmacies). Patients can obtain their own DCC via the zVEM Patient Portal (https://zvem.ezdrav.si) or the zVEM Mobile Application. The digital solution is located among the core eHealth services and contains the building blocks and functional components depicted in Figure 1.

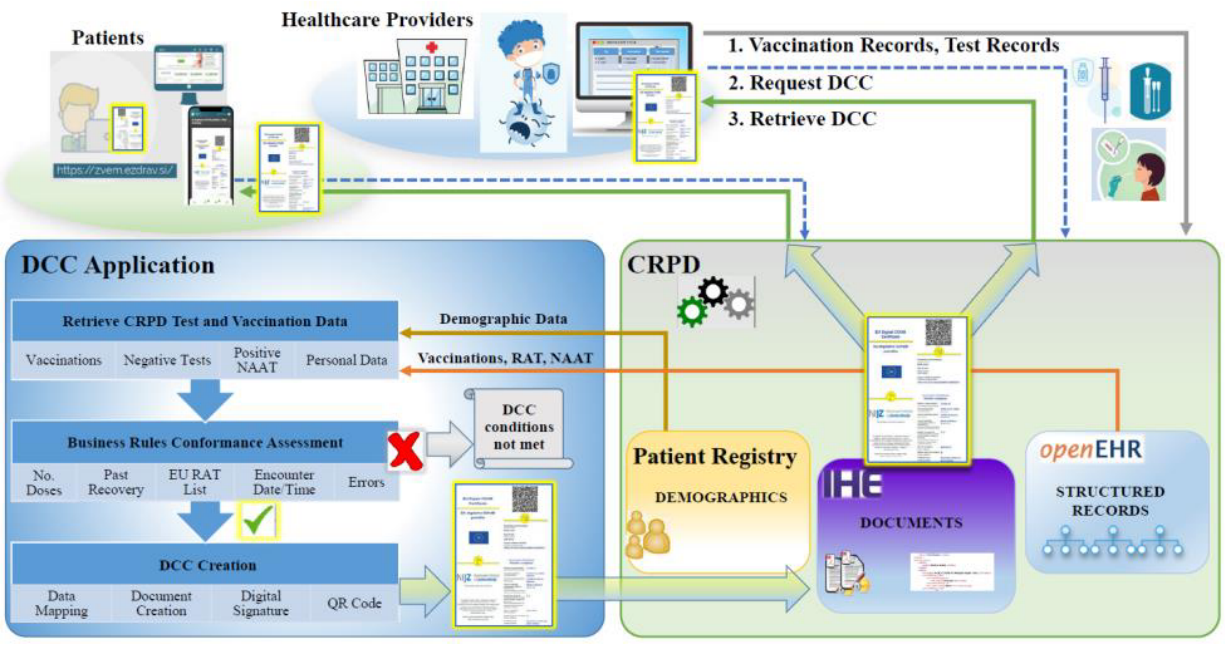

Figure 1. The DCC system architecture.

The DCC Application ensures the retrieval of data from the CRPD and its conversion in accordance with the EU standards, the verification of data conformance with the rules for issuing the DCC, and the creation of the DCC with a digital signature and QR code. High performance and dedicated data queries of vaccination and COVID-19 test result records extract the current data that are relevant for the issuance of the DCC. Queries are separate from the general CRPD services, which ensures sufficient responsiveness with a minimal load on the basic building blocks. The creation of a DCC is automatically triggered each time a CRPD query is made. For end users, the process of retrieving the DCC is exactly the same as for other documents. The DCC is associated with other documents available to patients and healthcare professionals, which is a great advantage. There are four types of DCC available in the CRPD: a DCC of a completed vaccination, a DCC of a negative NAAT, a DCC of a negative RAT, and a DCC of recovery from COVID-19. Only tests from the common list approved by the European Commission allow the issuance of a DCC. In the event of non-compliance with the required criteria, an error or failure to meet the DCC retrieval criteria is displayed on the document.

A prerequisite for the creation of a DCC is properly collected and recorded data. Healthcare providers must correctly submit records of tests and vaccinations to the CRPD. Providers of the RAT must properly report the testing results, and accredited 
microbiological laboratories must provide NAAT results. As a rule, laboratories also provide the original microbiological report in pdf format. Records can be submitted through local information systems to the Patient Summary or the Electronic Vaccination Registry. The query for a DCC is triggered in the same way as for other documents in the CRPD. When a user of the zVEM Patient Portal or a local information system at a healthcare provider initiates a query for a DCC, the DCC Application is automatically called. The DCC Application obtains data from the CRPD and, in accordance with the rules, creates the DCC based on the data currently available (vaccinations, a DCC of the last negative test in the past 8 days, a DCC of a positive NAAT in the last 180 days). In accordance with the technical rules, the document is signed in the DCC Application with a valid system certificate issued by the NIJZ. An essential component of the DCC is the QR code, which enables authorized personnel to conduct automatic verification. The DCC Mobile Verification Application reads the data from the QR code, and verifies the authenticity and validity of the DCC. If all conditions are met and all business rules are followed, the DCC is available to the user; if not, an error or failure to meet the DCC retrieval criteria is displayed on the document.

\section{Discussion}

Slovenia successfully introduced the DCC even before the deadline of 1 July 2021. However, the whole project - which was directed by the European Commission, which provided technical guidelines and the central digital platform (EU Gateway) required for the issuance and verification of the DCC - was managed awkwardly and inconsistently in some parts. This is somewhat understandable, as it was necessary to find the lowest common denominator of the EU Member States, EU institutions, and national and international healthcare organizations. Despite the lengthy harmonization process, the EU Member States have retained some autonomy in setting national rules for crossing their state borders, which further complicates the establishment of uniform DCC solutions at the EU level and brings about considerable confusion among citizens, who are not adequately informed of the fast-changing rules.

The whole DCC concept is relatively complex due to the multiple and rather dispersed data sources, on one hand [5], and the quite complicated and numerous business rules, on the other. If we disregard the pressure from all sides and the frantic circumstances in which the project was implemented [6], it should be noted that the latter factors caused the most problems in the development of the DCC solution and its introduction into everyday use. The lack of qualified digital certificates required to retrieve the DCC via the zVEM Patient Portal, the poor quality of the captured data, missing data or incomplete reporting of data on vaccinations, recovery, and testing by healthcare providers, legal issues - including data security and protection, rapid changes in code lists (especially the valid RATs on the EU list), problems with citizens who have recovered from COVID-19 or have been vaccinated or tested abroad, the lack of relevant data on foreigners living in Slovenia, and dilemmas in formulating and complying with the business rules due to unpredicted combinations of vaccines, tests, and time frames, are some of the key issues that Slovenia encountered in introducing the DCC solution. As elsewhere, delayed actions due to the coordination of policymakers and other stakeholders (national and EU), the lack of awareness of citizens, inadequate and inconsistent communication, and the misleading information that has flooded the media space, have only exacerbated the above problems. Nonetheless, approximately 990,000 
distinct DCCs have been issued in Slovenia (the vast majority sent by post), which is a rather high number in terms of the population ( 2.1 million) and the share of those fully vaccinated, which is $42 \%$. The Slovenian DCC application enables easy modifications of business logics without the need to upgrade the core components. Moreover, connection to the national CRPD platform guarantees the highest level of both reliability and inclusion. The central DCC service is ensuring medical-grade quality, and the DCC is available to the entire population. Operational experience has proven that the Slovenian DCC architecture is providing the required resilience, flexibility, and responsiveness. According to the available data reported by the EU Member States [7], Slovenia is among the countries with the highest number of DCC issued per capita.

However, the effectiveness of limiting the spread of COVID-19 infections by means of the DCC and related approaches has yet to be evaluated [8], and the wider implications of similar measures is an issue that future research in this area should address.

\section{Conclusions}

The establishment of political consensus and the construction of central digital platforms at the EU level, with well-defined protocols and rules for the integration of Member States, could be a major advantage in the future development of cross-border eHealth services, which will hopefully not be associated only with pandemic-related challenges.

Nevertheless, as the experience with the DCC has revealed, all such EU projects require well-developed national infrastructure, an appropriate architecture of national healthcare digital solutions and databases, a sufficient number of competent experts, precise rules for processing data, and, of course, sufficient funding and a suitable legal framework. Without awareness thereof, such projects can quickly turn into unproductive improvisation that fails to address the needs of EU citizens and cannot contribute to achieving the declared public health objectives.

\section{References}

[1] Regulation (EU) 2021/953 of the European Parliament and of the Council of 14 June 2021 on a framework for the issuance, verification and acceptance of interoperable COVID-19 vaccination, test and recovery certificates (EU Digital COVID Certificate) to facilitate free movement during the COVID-19 pandemic. Brussels, 14.6.2021.

[2] Chen LH, Petersen E, Blumberg L, Piyaphanee W, Steffen R. COVID-19 health passes: Current status and prospects for a global approach. J Travel Med. 2021 Aug 3;taab118.

[3] Thomas G. How to do your case study. Thousand Oaks, CA: Sage Publications; 2021.

[4] Lindgren BM, Lundman B, Graneheim UH. Abstraction and interpretation during the qualitative content analysis process. Int J Nurs Stud. 2020 Aug;108:103632.

[5] Mbunge E, Dzinamarira T, Fashoto SG, Batani J. Emerging technologies and COVID-19 digital vaccination certificates and passports. Public Health Pract. 2021 Nov;2:100136.

[6] Cardenas NC. Advancing strategic policy on European Union digital COVID-19 certificate. J Public Health. 2021 Jun;fdab227.

[7] European Commission. eHealth Network. Statistics and reporting. Brussels, 24.9.2021.

[8] Mehtar S, Preiser W, Lakhe NA, Bousso A, TamFum JJM, Kallay O, Seydi M, Zumla A, Nachega JB. Limiting the spread of COVID-19 in Africa: one size mitigation strategies do not fit all countries. Lancet Glob Health. 2020 Jul;8(7):e881-e883. 